\title{
Quality and safety of emergency care in inpatient facilities in Russia
}

\author{
Igor ivanov, Oleg shvabskii, Ildar minulin and Aleksandra shcheblykina*
}

FSBI "Center for Monitoring and Clinical and Economic Expert Evaluation" of Federal Service for Surveillance in Healthcare - Moscow, Russia

\begin{abstract}
Goals: This study aimed to analyze the results of external audits in respect of the "Emergency care in inpatient facilities" section in medical facilities in Russia.

Design: Analysis of the results of audits in medical facilities in Russia.

Setting: 30 medical facilities implementing Recommendations.

Results: Nowadays, there are complex and structural problems of emergency care's delivery management in Russian medical facilities. The resulting level of conformity of medical facilities to the requirements of the "Emergency care in inpatient facilities" section of Practical Guidelines (Recommendations) on the internal system of quality and safety control of medical care in medical facilities was $42.5 \%$. Only in 3 of the 30 medical facilities in question, the level of conformity of the emergency care delivery system to the requirements of the Guidelines was over 80\%, which highlights the effectiveness and safety of this system. 21 of the 30 medical facilities in question shows a critical level of conformity $(<50 \%)$ to the requirements of the Guidelines.
\end{abstract}

\section{Introduction}

The issues of quality and safety in healthcare are becoming high on the agenda in Russia. One understands the importance of implementation of objectively effective quality management systems in accordance with national standards for medical practice management.

Russian legislation in the sphere of healthcare provides for three levels of quality and safety control of medical care: state control, institutional control, and internal control [1]. The main form of quality control in medical facilities is the internal system of quality and safety control of medical care, which is the Russian legislation's requirement for licensing of the medical care.

It is also legislated that the chief executive of a medical facility determines the procedure for the internal quality and safety control of medical care in a medical facility, and it became a problem associated with the absence of the unified approach to the management of the quality and safety control of medical care in Russia.

In 2015, Federal State Budgetary Institution "Center for Monitoring and Clinical and Economic Expert Evaluation" of Federal Service for Surveillance in Healthcare (FSBI CMCEE at Roszdravnadzor) developed Practical Guidelines (Recommendations) on the internal system of quality and safety control of medical care in medical facilities [2]. These Guidelines became the prototype of the national safety and quality healthcare standard for hospitals in Russia. The Guidelines were developed with due consideration of the requirements of current worlds standards: Joint Commission International Standards for Hospital (USA), National Safety and Quality Health Service Standards (Australia), Canadian Council on Health Services Accreditation (Canada), and others. The Guidelines provided the basis for the System of the voluntary certification of medical facilities "Quality and Safety of Medical Care" (Figure 1), which was registered in 2016 [3]. The certification applies by evaluation of medical facility based on the requirements of the Guidelines.

\section{The Guidelines include the following main fields of concern:}

1. Human resources management.

2. Patient Identification;

3. Epidemiologic safety. Preventing and Controlling Healthcare Associated Infections;

4. Drug safety. Pharmacovigilance;

5. Control of quality and safety of medical devices circulation;

6. Emergency care in inpatient facilities.

7. Managing clinical responsibility. Patient internal and external transfer;

8. Surgical safety. Preventions of risks associated with surgical intervention;

9. Blood management;

10. Safe environment for the delivery of care;

11. Patient care management. Preventing and managing falls, pressure injuries;

*Correspondence to: Aleksandra shcheblykina, 1FSBI "Center for Monitoring and Clinical and Economic Expert Evaluation" of Federal Service for Surveillance in Healthcare - Moscow, Russia, E-mail: a.shchebl@gmail.com

Key words: emergency care, Russia, audit, quality and safety, quality management system

Received: June 14, 2018; Accepted: June 26, 2018; Published: June 29, 2018 


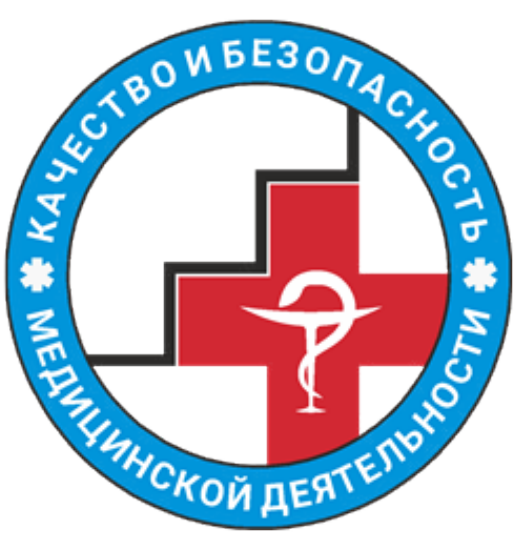

Figure 1. Flow chart. SAMU: Service d'aide médicale d'urgence, qSOFA: quick sepsisrelated organ dysfunction assessment score, ICU: intensive care unit; ED: emergency department. MICU: mobile intensive care unit, EMT: emergency mobile team.

12. Evidence-based healthcare. Compliance with clinical guidelines (Protocols).

Audit is the form of evaluation of the conformity of the medical facility to the requirements of the Guidelines $[4,5]$. The audit is effective method for evaluation and revealing of existing problems. Trained specialists from the personnel of a medical facility conduct the internal audit, or self-evaluation; specialists from a separate independent organization carry out the external audit.

\section{Methods}

The study includes the evaluation of medical facilities regarding their compliance with the requirements of the "Emergency care in inpatient facilities" section of Roszdravnadzor's Practical guidelines (Recommendations) on the internal system of quality and safety control of medical care in medical facilities.

The following groups of factors assessed in this section:

1. The health service organization in Emergency Department. System of emergency care in a medical facility;

2. Using of emergency care algorithms (for a medical facility);

3. Sorting emergency patients;

4. The timeliness of health service delivery;

5. The system of emergency notification/gathering of the stuff;

6. Personnel call system for patients;

7. The readiness of a medical facility to deliver healthcare within emergency situations;

8. Maintaining of equipment and toolkits (sets) for emergency care delivery;

9. Availability of support services (laboratory, instrumental diagnostics) 24 hours per day, 7 days per week, 365 days per year;

10. The readiness of the personnel to deliver emergency care. Training for the personnel;

11.Informing patients, patient feedback;

12. Adherence to the principles of confidentiality and privacy during appointment and healthcare delivery.
The methodology of conducting the study assumes the calculation of the overall index of the conformity of the medical facilities to the "Emergency care in inpatient facilities" section. The assessment sheet for this section includes the list of criteria combined into groups. The assessment system is binary; it determines the conformity or nonconformity to one or another criterion. The non-conformity to any criterion in the group is the reason to consider the whole group of parameters non-conforming.

The sources of information described on the Figure 2.

The article uses the results of 30 audits of medical facilities, which are super specialty hospitals that deliver both elective and emergency care including high-tech medical care. The average hospital bed capacity was 500 beds (up to 1000), the average number of the personnel (both medical and non-medical professionals) was about 2000 people in each facility. The mentioned medical facilities' structure included regional or primary vascular centers and at least two intensive care units.

Initiators of conducting audits were the authorities of medical facilities. All the members of expert teams adhered to the principles of confidentiality and goodwill. Experts made a point of the fact that the authorities of the medical facilities in question had ensured the personnel that they would not be punished after the audit in any case which made the personnel more open. According to the conditions of the agreement between medical facilities and FSBI CMCEE at Roszdravnadzor the experts had access to all the rooms of the facilities and to all medical and organizational records.

The study was carried out in compliance with ethical standards, taking into account measures for protection of the study subjects' privacy and the privacy of their personal data. Before interviewing patients, voluntary verbal informed consent statements were received within the framework of the study conducted: the study subjects were informed about purposes, methods, any possible conflicts of interest, expected results, potential risks, and any other important aspects of the study, the subjects also gave their consent to the fact that the received data would be used for research purposes. Before the interview the participants were informed that, the received information would be used without mentioning their names and the names of facilities.

\section{Results}

The health service organization and emergency care delivery is vital for any inpatient facility regardless of a medical facility's size and the level of care delivery. The final result depends largely on timeliness, effectiveness and safety of care delivered at the earliest possible time after admission a patient to hospital.

After the audits conducted, the resulting level of conformity of medical facilities to the requirements of the "Emergency care in inpatient facilities" section was $42.5 \%$.

Only in 3 of the 30 medical facilities in question the level of conformity of the emergency care delivery system to the requirements

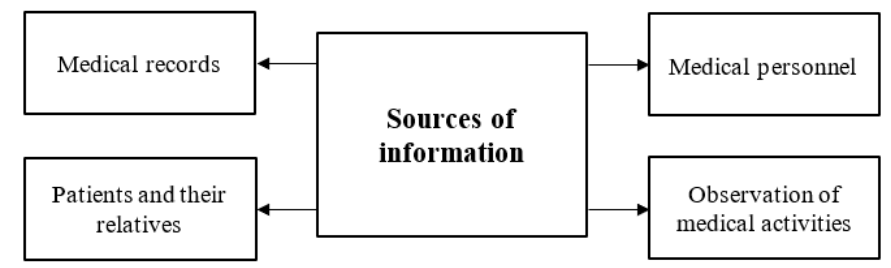

Figure 2. The Sources of Information. 
of the Guidelines was over $80 \%$, which highlights the effectiveness and safety of this system.

It should be noted that more than a half (21) of the medical facilities in question shows a critical level of conformity $(<50 \%)$ to the requirements of the Guidelines (Figure 3).

Emergency care requires teamwork of the whole staff, both healthcare professionals and support services, unfailing operation of equipment, availability of all the necessary drugs, and so on. The results of the assessment of conformity of medical facilities to the requirements of the "Emergency care in inpatient facilities" section are shown in Figure 4.

The group of parameters 1 contains the criteria, which define organizational aspects of system of emergency care in a medical facility:

- The procedure of the management of the hospital emergency rooms work;

- The procedure of the management of emergency care delivery;

- Responsible executives, the composition of specialized commissions;
- The composition and functioning procedure of a multidisciplinary work group on the management of emergency care delivery;

- Audits of the emergency care delivery system and others.

Only in 5 of the 30 medical facilities in question adhere to the specified requirements. Most of the hospitals studied had no comprehensive system to audit medical performance. Only four conducted audits on a regular basis.

Algorithms of emergency care delivery (2) (especially in case of cardio-pulmonary resuscitation and anaphylactic shock) do not conform to the Russian and international clinical guidelines in 26 of the 30 medical facilities in question. The use of unified algorithms developed in accordance with peculiarities of a medical facility, which may help in high-quality emergency care delivery when necessary, makes the process of healthcare delivery more controllable.

Only 9 of the medical facilities in question had a properly functioning personnel call system (6) which is maintained on a regular basis. Often only common premises are equipped with personnel call systems, and patients in critical conditions have no physical ability to call a nurse when they need help.

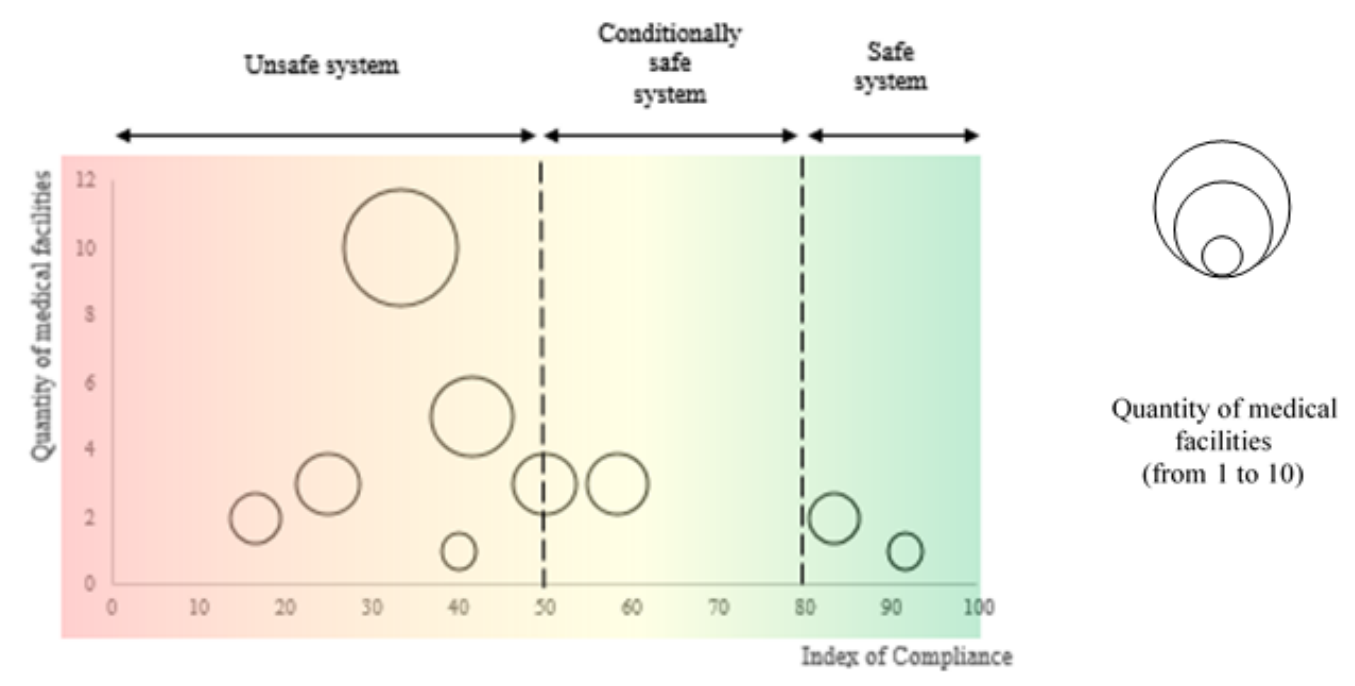

Figure 3. Critical level of conformity $(<50 \%)$ to the requirements of the Guidelines.

25

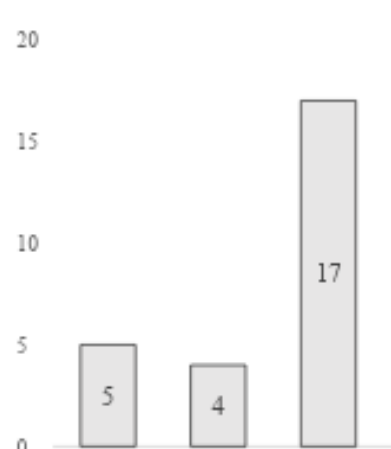

(1)
(2)
(3)

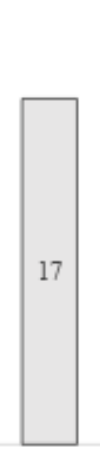

(4)

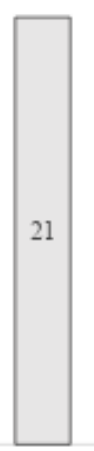

(5)

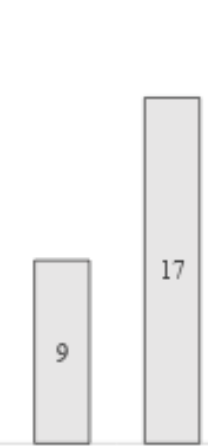

(6)
(7)

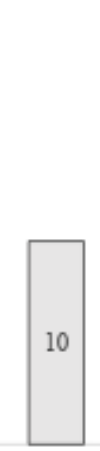

(8)

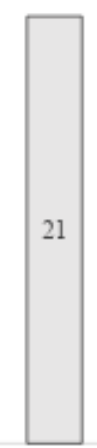

(9)

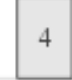

(10)

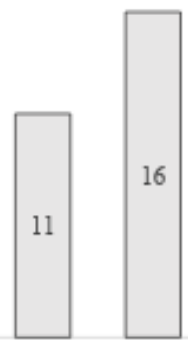

(11)
(12)

Figure 4. Emergency care in inpatient facilities. 
The toolkits (sets) and equipment for emergency use (8) are maintained on a regular basis only in 10 of the 30 medical facilities studied. Often there are sets for anaphylactic shock treatment in a hospital, but they contain algorithms, medical devices and drugs, which do not conform neither to the current Russian legislative requirements nor to international clinical guidelines. There are no operation monitoring systems for the equipment used for emergency care delivery. Though, it is essential to conduct systematic monitoring of their condition, actualize the equipment, and train the personnel properly, - in this case only the sets for emergency care delivery are used effectively.

In 26 of the 30 medical facilities in question, there are serious problems with the assessment of practical readiness of the healthcare personnel to deliver emergency care and resuscitation (10): many healthcare professionals take guidance of the personnel of intensive care unit when such situations occur. There is a need to develop and implement programs of education and training personnel including the use of simulators as practical use of some knowledge on emergency care delivery is possible only in case of the practical experience.

The timeliness of the healthcare delivery (4) is often very important when a patient visits a hospital. Time standards of emergency care delivery are not adhered in 13 of the 30 medical facilities studied: standards for initial examination by a nurse, initial examination by a doctor, diagnosis, patient transfer to the department/operation room/intensive care unit, diagnostic studies, and other standards of healthcare delivery in accordance with clinical guidelines for certain conditions (ACS, Acute Cerebrovascular Event, and so on).

The medical facilities in question also showed non-conformity with the existing requirements in sorting patients depending on the severity of their condition and the need to deliver emergency care, the absence of educational trainings for the personnel to use proper algorithms in case of emergency notifications and gathering of the personnel, the procedure of routing patient streams in case of emergency situations, and time standards for carrying out laboratory assessment and instrumental diagnostics.
In 10 of the 30 medical facilities there is no proper provision of information for patients, their condition, diagnosis, therapies and so on are not explained to them properly; there is no proper patient feedback system for interviewing patients while they stay in a medical facility about quality and safety of the delivering care.

\section{Conclusion}

Thus, nowadays there are complex and structural problems of emergency care's delivery management in Russian medical facilities.

The example of the analysis of the audits carried out in 30 Russian inpatient facilities in terms of the "Emergency care in inpatient facilities" section shows, that the proposed Practical Guidelines (Recommendations) on the internal system of quality and safety control of medical care in medical facilities helps to reveal risks associated with emergency care delivery and to plan corrective actions.

Our practice shows that it is possible to implement in Russian medical facilities a complex system of medical care quality and safety management which includes the requirements of current world standards, is based on conducting audits on a regular basis, and uses comprehensive and process approaches and risk management and patient-oriented principles.

\section{References}

1. Federal Law (2011) On fundamental healthcare principles in the Russian Federation, N 323-FL.

2. Bulletin of Roszdravnadzor (2016) Proposals for arrangement of inner quality and safety control of medical activity in medical organization (hospital) 2: 35-36.

3. Ivanov IV, Shvabskii OR, Minulin IB, Shchesyul AG (2017) Medical activity: quality and safety. Standards and Quality 3: 72-74.

4. Ivanov IV, Shvabskii OR, Minulin IB, Emanuel A (2017) Audit as a tool of healthcare quality assessment. Standards and Quality 11: 27-29.

5. Ivanov IV, Shvabskii OR, Minulin IB, Shcheblykina AA (2018) Results of audits of quality and safety of medical activity in hospital. Qual Manag Healthcare 1: 18-22.

Copyright: (C2018 Ivanov I. This is an open-access article distributed under the terms of the Creative Commons Attribution License, which permits unrestricted use, distribution, and reproduction in any medium, provided the original author and source are credited. 